\title{
A Study on Microbial Profile of Corneal Ulcer Cases in a Tertiary Health Care Centre of Southern Assam
}

\author{
Barnamoy Bhattacharjee ${ }^{1}$, Atanu Chakravarty ${ }^{2}$ \\ ${ }^{1}$ Post Graduate Trainee in Microbiology (M.D. Microbiology), Silchar Medical College \& Hospital, Assam \\ ${ }^{2}$ Assistant Professor, Department of Microbiology, Silchar Medical College \& Hospital, Assam \\ Corresponding Author: Barnamoy Bhattacharjee
}

\begin{abstract}
Background-Visual impairment due to corneal ulceration has for long been acknowledged as leading cause of blindness both worldwide and in India only next to Cataract. The aetiology for infective corneal ulcer varies considerably with occupation of patients in different regions of India. Thus, continuing with empirical management without lab diagnosis leads to protracted clinical course. So, understanding the microbial profile of corneal ulcers in agriculture dependent population of Southern Assam helps in improved management of this morbidity.
\end{abstract}

Aims- To 1) Find the prevalence of infected corneal ulcers in untreated patients attending Ophthalmology department of a tertiary care hospital of Southern Assam. 2) Study the pattern of microbial aetiology in the infected corneal ulcer cases. 3) Find the association of infected corneal ulcers with various attributing factors.

Method-Corneal scrapings from 86 routine untreated cases of corneal ulceration from Ophthalmology Department were studied prospectively from Dec2017 to Jan2019 and subjected to Gram staining and direct examination with $10 \% \mathrm{KOH}$. Culture on SDA and Blood Agar were incubated at $25^{\circ} \mathrm{C}$ and $37^{\circ} \mathrm{C}$ respectively and followed up for 3 weeks before declaring sterile. Antimicrobial sensitivity was performed for bacterial isolate and yeast-like species. Slide culture and LPCB tease mount were done for identification of moulds.

Result- Out of the 86 cases, 58 were positive in direct microscopy, of which 44 were culture positive. Of the 28 samples negative in direct microscopy, 2 were positive by Culture. Bacterial isolates were found in 5 cases and fungal aetiology in 41 cases.
Conclusion- The prevalence of infected corneal ulcer as per standard of Culture positivity is $53.5 \%$ (46/86). Mycotic involvement was found in $90 \%$ cases. Important fungal isolates identified were Aspergillus species, Penicillium species, Curvularia species, Fusarium species, Sarocladium species. Higher prevalence was significantly associated with harvesting season, agricultural practice and history of trauma while diabetes and steroid intake were not significantly associated with mycotic ulcers.

Key Words- Corneal ulcer, Assam, Fungal, Fusarium, Sarocladium, Pseudomonas

\section{INTRODUCTION}

Corneal infection is a leading cause of ocular morbidity and blindness worldwide ${ }^{[1]}$ and Microbial keratitis is one of the predominant causes of preventable form of blindness. ${ }^{[2]}$ Visual impairment and subsequent blindness due to microbial keratitis followed by corneal ulceration has for long been acknowledged as leading cause of blindness both worldwide and India only next to Cataract. Corneal blindness is responsible for 1.5-2 million new cases of monocular blindness every year. ${ }^{[3]}$ In India, approximately 6.8 million people are suffering from corneal blindness. Out of these, about 1 million have bilateral corneal blindness. It has been estimated that the number of people afflicted from corneal blindness in India will increase to 10.6 million by $2020 .{ }^{[4]}$ There is a significant variation in the prevalence of corneal ulceration in different parts of the world. This can be attributed to difference in 
climatic conditions, difference in occupation as well as other socioeconomic factors. ${ }^{[1]}$ The causative organism responsible for infective corneal ulcer varies considerably by region. In the Western population, viral corneal infections account for the majority of cases of corneal blindness. On the other hand, fungal and bacterial infections of the cornea predominate in the Asian subcontinent. ${ }^{[5]}$ Fungi are the most common etiological agents which account for 30$40 \%$ whereas bacteria account for $13-48 \%$ of all cases of suppurative keratitis. [6] Various risk factors have been implicated for increased incidence of fungal keratitis including widespread use of antibiotics and steroids, use of contact lenses, and postoperative infections. ${ }^{[7]}$ Being in the subtropical region, the north-eastern part of India has conducive environmental conditions like high rainfall, longer rainy season, and high humidity throughout the year for fungal growth. Moreover, the majority of the rural population in Southern part of Assam is engaged in agriculture (cereals and vegetables) including the labour-intensive tea industry and contribute to Agrarian economy of the valley. This makes them more vulnerable to ocular trauma due to vegetative matter like paddy leaves and bamboo sticks and subsequent microbial infection and corneal ulceration. Since the underlying microbiological aetiology of infective corneal ulcer shows a wide regional variation, continuing with empirical management in presence of precipitating factors like ocular trauma, diabetes mellitus without timely establishing the aetiology by lab diagnosis may lead to protracted clinical course of keratitis and ulceration therefore leading to dimness of vision and ultimately blindness. Therefore, understanding and awareness of the clinicians about the clinical and microbial profile of corneal ulcers in a predominantly agriculture dependent population of Southern Assam helps in improved management of this sight-threatening condition.

Thus, this study has been undertaken to
1) Find the prevalence of infected corneal ulcer cases in untreated patients attending Ophthalmology department of a tertiary care hospital of Southern Assam.

2) To study the pattern of microbial aetiology in the infected corneal ulcer cases.

3) To find the association of infected corneal ulcers with various attributing factors.

\section{MATERIALS AND METHOD}

This prospective cross-sectional study was carried out from December 2017 to February 2019 on 86 clinically suspected routine untreated cases (of all age) of corneal ulceration including cases from both gender and all ages attending the Ophthalmology OPD of the only tertiary health care centre of Southern Assam which also sees foot fall of patients from neighbouring north-eastern states of Mizoram, Tripura, Meghalaya and Nagaland. Complete personal and medical history and presenting features of the cases were noted. Cases empirically treated with topical antibacterial and antifungal drops were excluded from this study. Scrap materials were collected from base and edges of the keratic ulcers by Bard Parker blade no-15 and one part of the collected material was spread on 2 clean sterile grease free labelled glass slides and the other part of the scrapped material was stabbed in the shape of letter $\mathrm{C}$ or letter $\mathrm{S}$ in Blood Agar and Sabouraud's Dextrose Agar with Cycloheximide and Chloramphenicol supplied by HiMedia. The direct smear examination of the scrap material on glass slide was performed by $10 \% \mathrm{KOH}$ mount to look for fungal elements and Gram's staining was performed to detect presence of both bacterial pathogen and Fungal elements. The inoculated plates of Blood Agar and Sabouraud's Dextrose agar were incubated at $25^{\circ} \mathrm{C}$ and $37^{\circ} \mathrm{C}$ in two sets and followed up twice weekly for 3 weeks to detect fungal growth before declaring those sterile. Culture plates showing positive 
growth were inspected for colony characteristics especially colour, surface, texture, margins, sporulation on obverse and pigmentation on reverse in case of mycelial growth. Lactophenol Cotton Blue tease mount examination was performed to identify the fungal growth. In cases where plate culture could not decisively identify the fungal pathogen Slide culture was performed on Corn Meal Agar or Potato Dextrose Agar to identify the pattern of characteristic microconidia of filamentous fungi. Bacterial isolates were identified by colony characteristics, gram staining and routine biochemical tests and anti-microbial sensitivity testing was done on Mueller Hinton agar in accordance with CLSI guidelines. Yeast like species was identified by germ tube test, colour of growth on CHROM agar etc and antifungal sensitivity testing was done on Mueller Hinton Agar with Methylene blue supplied by HiMedia.

Statistical Calculation- It was done by using online software in Socscistatistics.com. $P$ value was determined from Chi square score using $2 \times 2$ contingency table for each attributing factor. Significance level was set at $p$ value $\leq 0.05$.

\section{RESULT}

Out of the total 86 cases studied, 58 samples were positive in Direct Microscopy and out of which 44 showed growth in Culture. And of the 28 samples which were negative in Direct Microscopy, 2 cases were confirmed by culture after ruling out Laboratory contamination. Thus, taking Culture positivity as Gold standard, the prevalence of infected corneal ulcers came up as $53.5 \%$. Agricultural practice was found to be significantly associated with higher no of infected corneal ulcer cases (Chi square value-9.75, $\mathrm{p}$ value-0.001788). Harvesting season also witnessed statistically significant higher no of infected corneal ulcer cases. (Chi square 11.6061, p value 0.000657.) Diabetes mellitus was not found to be significantly associated with mycotic corneal ulcer (Chi square value 0.012 and $p$ value 0.912695 .)
Table 1: OCCUPATIONAL DISTRIBUTION OF CASES

\begin{tabular}{|l|c|c|}
\hline Occupation & $\begin{array}{l}\text { Total cases } \\
\text { studied }\end{array}$ & $\begin{array}{l}\text { No. of culture positive } \\
\text { cases }\end{array}$ \\
\hline $\begin{array}{l}\text { Agricultural } \\
\text { practice }\end{array}$ & 38 & 28 \\
\hline Manual Labourer & 28 & 12 \\
\hline Others & 20 & 06 \\
\hline
\end{tabular}

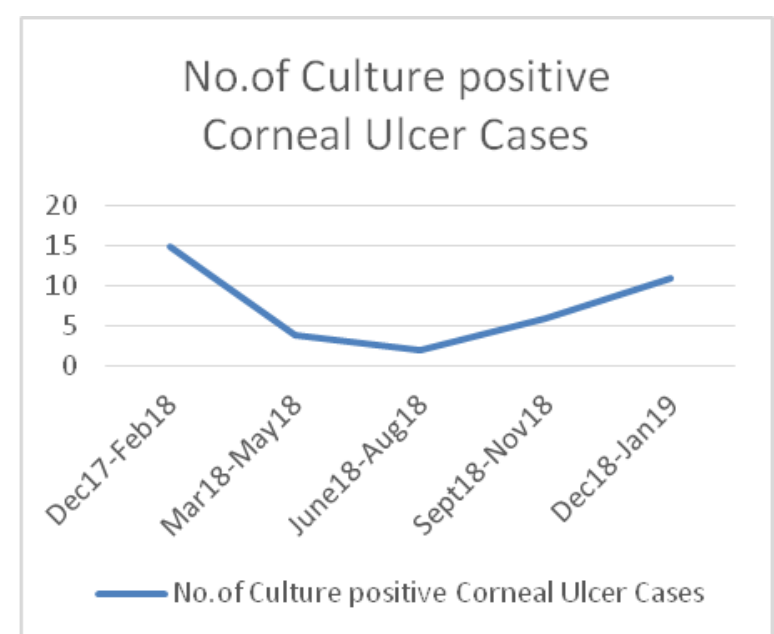

Graph-1: Seasonal distribution of Corneal Ulcer cases.
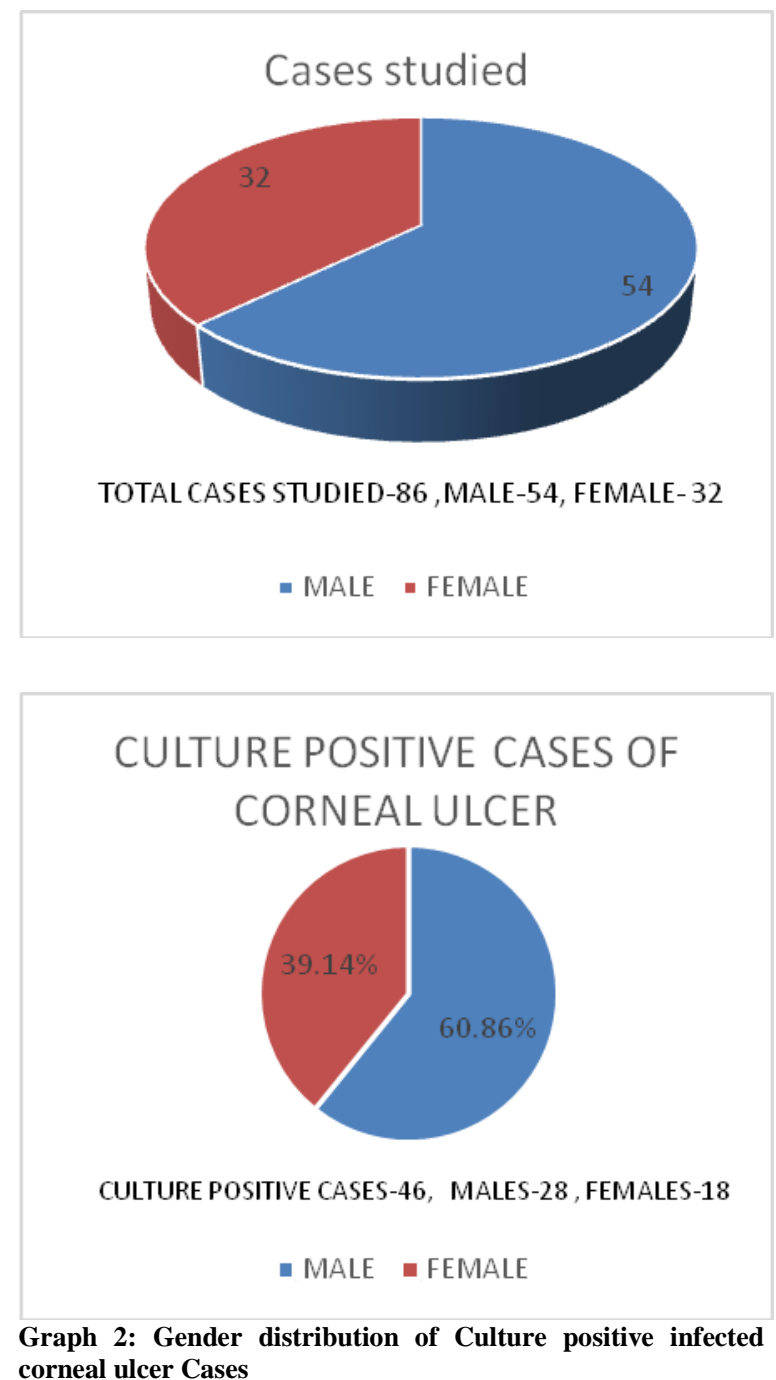

Graph 2: Gender distribution of Culture positive infected corneal ulcer Cases 
Table: 2- Result of Direct Microscopic Examination of Smear from Corneal Scrap

\begin{tabular}{|l|l|l|}
\hline $\begin{array}{l}\text { Direct Microscopy Positive } \\
\text { Samples }\end{array}$ & 58 & Culture Positive-44 \\
\cline { 3 - 4 } $\begin{array}{l}\text { Direct microscopy Negative } \\
\text { Samples }\end{array}$ & \multirow{2}{*}{28} & Culture Negative-14 \\
\cline { 3 - 3 } & & Culture Positive- 02. \\
\hline
\end{tabular}

Table 3: Mycotic Corneal Ulcers and Diabetes mellitus

\begin{tabular}{|l|c|c|}
\hline & $\begin{array}{l}\text { No. of Mycotic } \\
\text { Corneal ulcer cases }\end{array}$ & $\begin{array}{l}\text { No. of Bacterial } \\
\text { corneal ulcers }\end{array}$ \\
\hline Diabetic Cases & 03 & 01 \\
\hline $\begin{array}{l}\text { Non- Diabetic } \\
\text { Cases }\end{array}$ & 38 & 04 \\
\hline
\end{tabular}

$4.35 \%$

\section{Percentage of Cases affected}

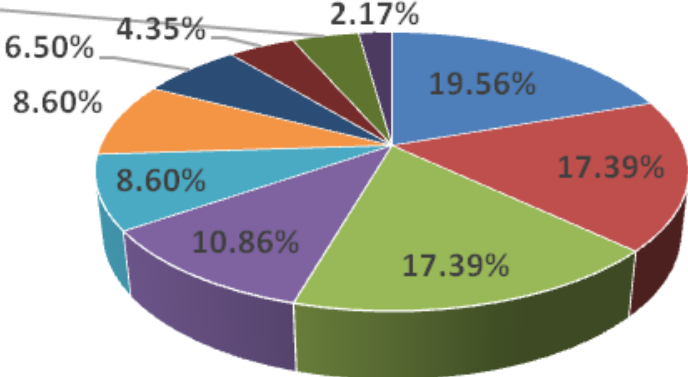

$$
\begin{array}{lll}
\text { - Aspergillusfumigatus } & \text { - Aspergillusflavus } & \text { - Fusarium species } \\
\text { - Curvularia species } & \text { - Sarocladium kiliense } & \text { - Penicillium species } \\
\text { - Pseudomonas aeruginosa } & \text { - Staphylococcus aureus } & \text { - Candida albicans }
\end{array}
$$

- Purpureocillium lilacinum

Graph 3: Distribution pattern of isolated pathogens from Culture positive Infected corneal Ulcer Cases

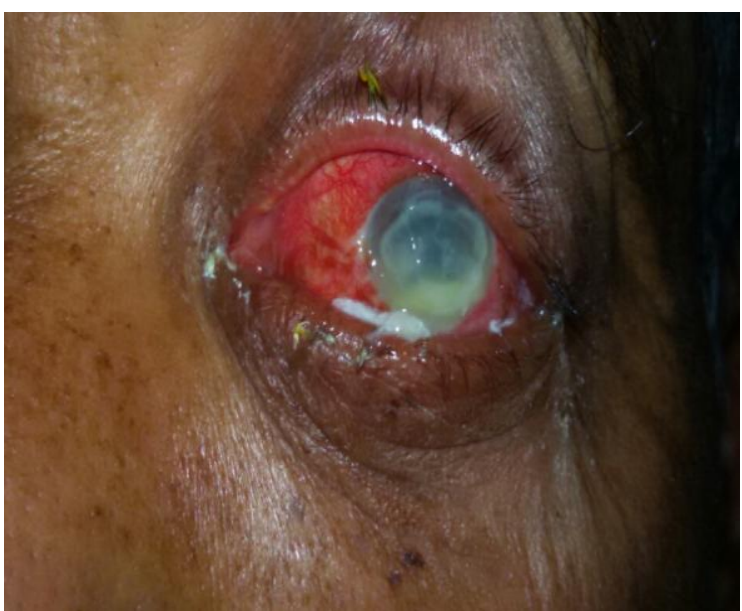

Hypopyon formation in an Infected corneal ulcer of left eye in an elderly male peasant.

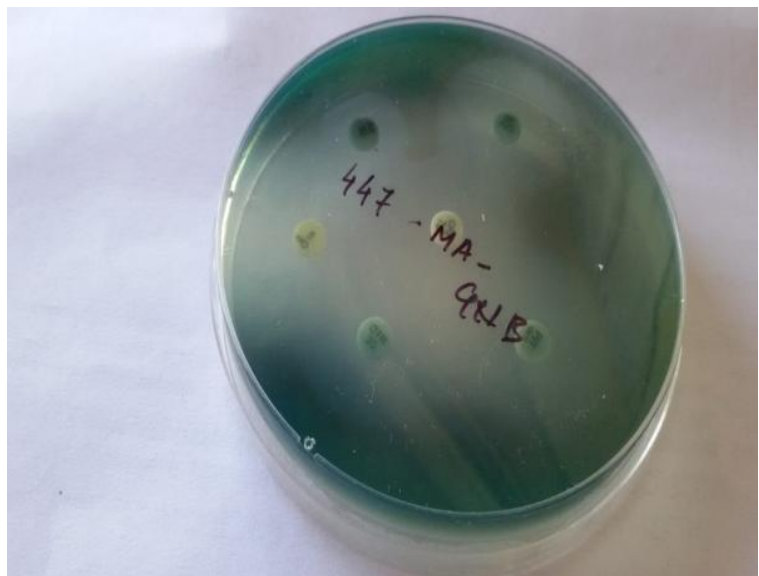

Antimicrobial sensitivity pattern of Pseudomonas aeruginosa on Mueller Hinton Agar

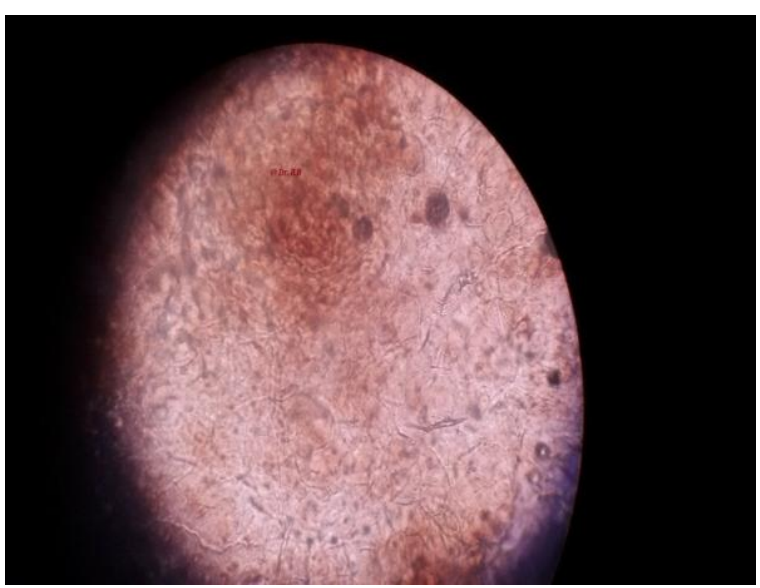

Plenty of Hyaline septate fungal hyphae with acute angled branching as seen in $\mathrm{KOH}$ mount examination of corneal scrap material under low power lens.

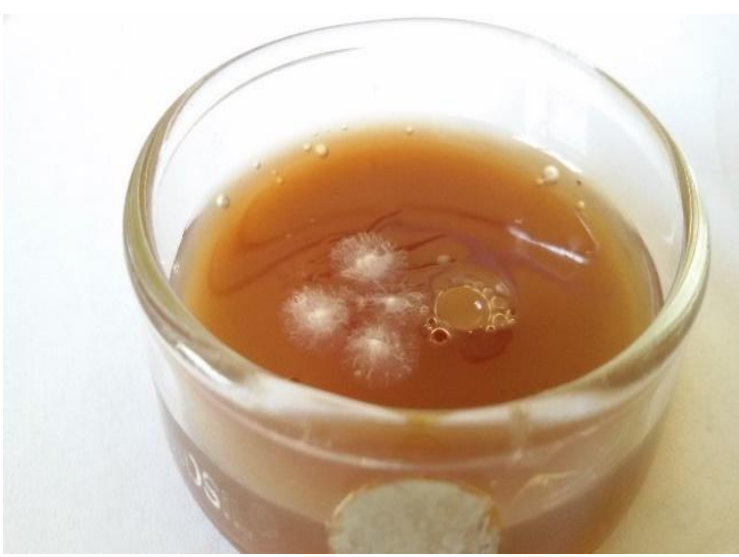

Fungal Colonies on Blood Agar plate 


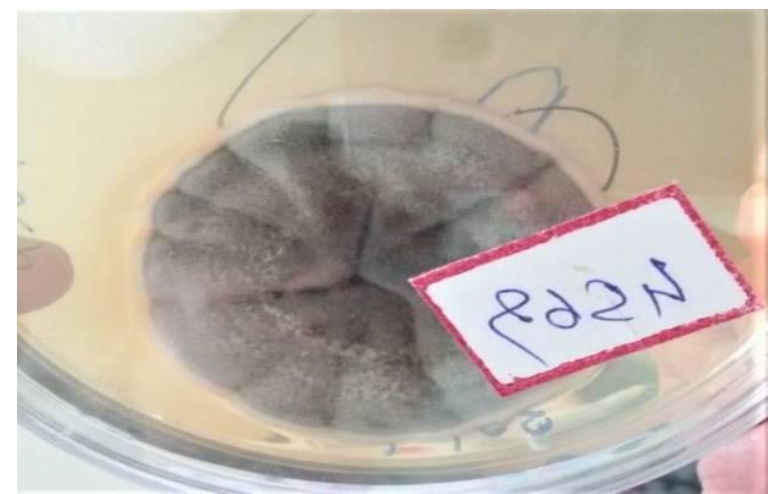

Colonies of Curvularia species on SDA plate

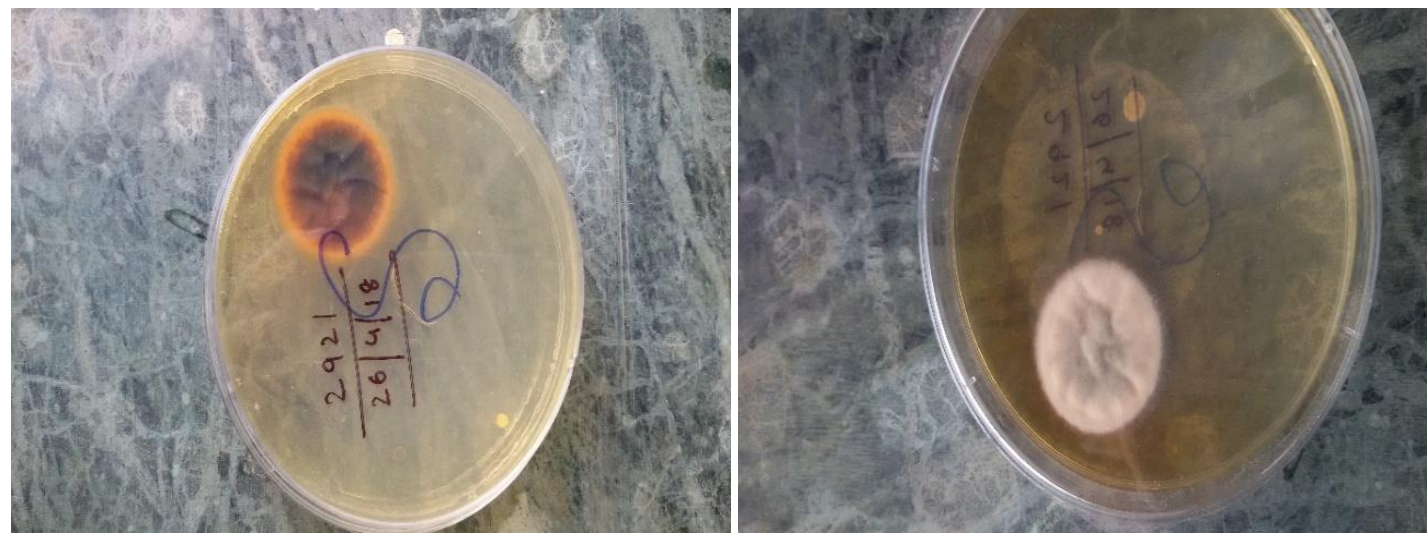

Reverse and obverse of colony of Fusarium species on Sabouraud's Dextrose Agar
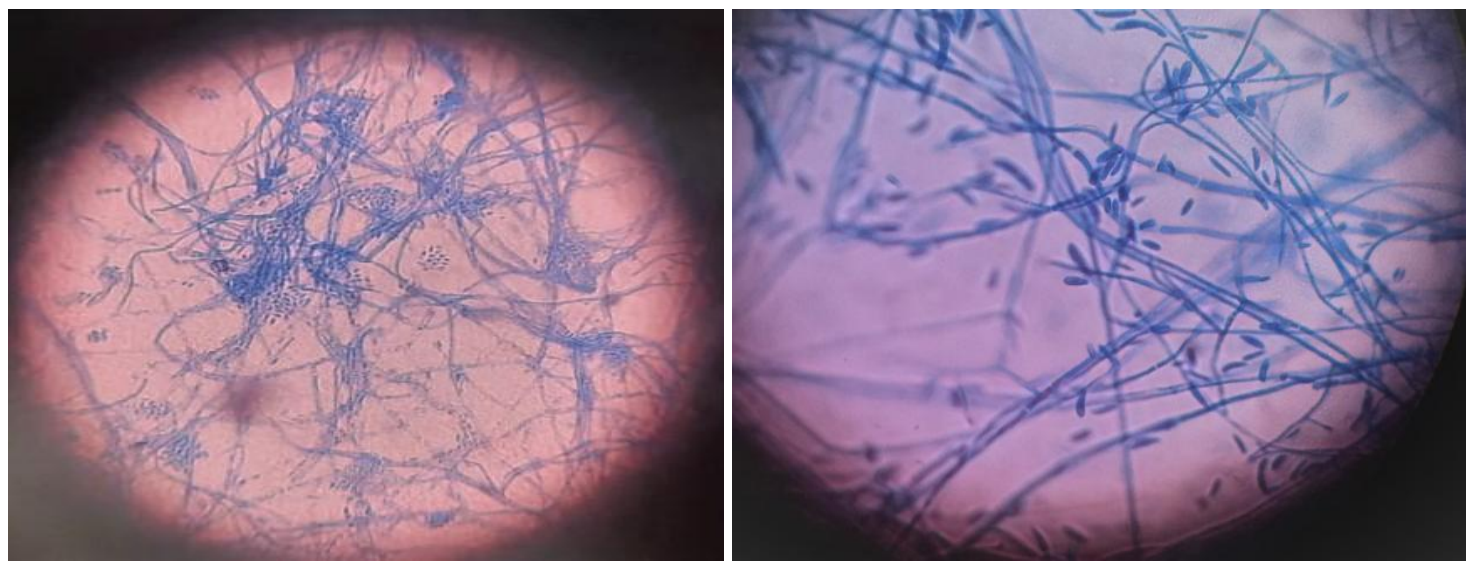

Lactophenol Cotton Blue mount from colonies on SDA plate culture shows microconidia suggestive of Fusarium species(left) and Sarocladium species (right)

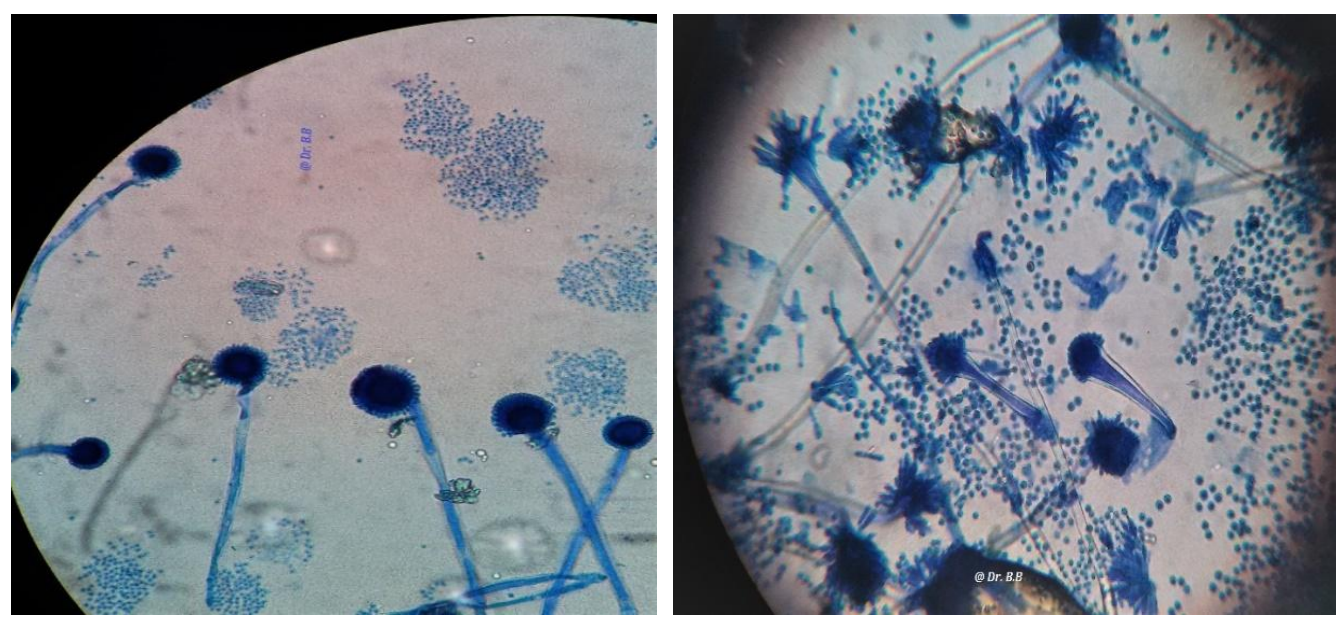

Conidiophores of Aspergillus flavus and Aspergillus fumigatus as seen in LPCB mount 


\section{DISCUSSION}

The prevalence of infected corneal ulcer in this tertiary care centre of Southern Assam which also caters to patients from neighbouring North-east Indian states as estimated from this microbiological study is $53.5 \%$. Almost Similar prevalence of $60 \%$ and $61 \%$ was found in Studies conducted in northern part of Assam by Reema Nath et al ${ }^{[8]}$ and Tapan Gogoi et al. ${ }^{[9]}$

In eastern India, prevalence of 50\% was found by Sanjeev Das and Jayashree Konar ${ }^{[10]}$ in a hospital-based study in West Bengal. In Gujarat of Western India, Rajesh Katara et al ${ }^{[11]}$ found $40 \%$ prevalence of infected corneal ulcers.

While $43 \%$ prevalence of infected corneal ulcer was found in Haryana of North India by Kanistha Sharma et al, ${ }^{[12]}$ it was found to be $54 \%$ by A Gupta et al. ${ }^{[13]}$ $62.66 \%$ prevalence of infected corneal ulcers was observed by C Senthilvadivu ${ }^{[14]}$ in his hospital-based study in Chennai and $72.5 \%$ culture positivity was found by Derek Y Kunimoto et al ${ }^{[1]}$ in elderly Corneal ulcer cases from Hyderabad. K Lakshmoji et al ${ }^{[15]}$ found $74 \%$ prevalence of infected corneal ulcer in a study conducted in rural population of Andhra Pradesh. But in contrast, a lower prevalence of $37.5 \%$ culture positive corneal ulcer cases was found in Karnataka by Chittur Y Ranjini et al $^{[16]}$

In Central India, the prevalence found by Shashi Gandhi et al [17] from Gwalior, Madhya Pradesh was 55\%. While in another study by Mehta et al ${ }^{[18]}$ in Ujjain Madhya Pradesh, the prevalence of culture positive infected corneal ulcer was found $71.6 \%$.

This study found Mycotic Keratitis to be the most common form of Corneal Ulcer infection which has been found to be concordant with observation of Nath et al, ${ }^{[8]}$ Tapan Gogoi et al, ${ }^{[9]}$ Rajesh Kataria et al, ${ }^{[11]}$ Kanishtha Sharma et al, ${ }^{[12]} \mathrm{K}$ Lakshmoji et al, ${ }^{[15]}$ Chittur Y Ranjini et al. ${ }^{[16]}$ But Derek Y kunimoto et al ${ }^{[1]}$ found bacterial pathogen to be the dominant aetiology behind infected corneal ulcers in elderly age group.

As observed by Rajesh Kataria et al, ${ }^{[11]}$ A Gupta et al, ${ }^{[13]}$ Gandhi S et al, ${ }^{[17]}$ in this study too, Aspergillus species infection was found to be the most common fungal aetiology followed by Fusarium species. But this is in discordance with records of Nath et al ${ }^{[8]}$ and Tapan Gogoi et al ${ }^{[9]}$ from Assam and Ranjini C Y et al ${ }^{[16]}$ et al who found Fusarium species to be the most common fungal pathogen for corneal ulcers followed by Aspergillus.

Pseudomonas aeruginosa was found as the commoner isolate in bacterial corneal ulcer cases which has been seconded by view of Rajesh Kataria et al ${ }^{[11]}$. But Tapan Gogoi et al, ${ }^{[9]}$ Sanjeev Das and Jayasree Konar, ${ }^{[10]} \mathrm{K}$ Lakshmoji et al, ${ }^{[15]} \mathrm{C}$ Senthil Vadidu ${ }^{[14]}$ Gandhi S et al. ${ }^{[17]}$ and Mehta et al ${ }^{[18]}$ found Staphylococcus aureus as the predominant bacterial isolate in the corneal ulcer cases while Staphylococcus epidermidis was the dominant pathogen in elderly corneal ulcer cases ${ }^{[1]}$.

While in this study higher numbers of cases of mycotic corneal ulcers were found during December to February corresponding to harvesting season in Assam, A Gupta et al found higher number of cases of mycotic corneal ulcer in post monsoon season (Sept to Nov). But Gandhi $S$ et al ${ }^{[17]}$ found maximum incidence of mycotic corneal ulcer cases in central India in the month of June and July.

The most common predisposing factor associated with infected corneal ulcer as revealed in this study was vegetative matter induced ocular trauma. This finding has been seconded by observation of Nath et al, ${ }^{[8]}$ Rajesh Kataria et al, ${ }^{[11]}$ A Gupta et al, ${ }^{[13]}$ C Senthil Vadidu, ${ }^{[14]} \mathrm{K}$ Lakshmoji et al, [15] Gandhi $\mathrm{S}$ et al, ${ }^{[17]}$ Mehta et al ${ }^{[18]}$. However, in contrast to this finding, in elderly age group the commonest predisposing factor for infected corneal ulcer found by Derek $\mathrm{Y}$ et al ${ }^{[1]}$ was previous ocular disease and ocular surgery.

Diabetes Mellitus was not found to be a significant risk factor for mycotic 
corneal ulcers in this study (Chi square score 0.012, $\mathrm{p}$ value 0.912). Similar observation has been made by Tapan Gogoi et al, ${ }^{[9]}$ Mehta et al ${ }^{[18]}$ where Diabetes mellitus was not found to be a significant risk factor for corneal ulceration. This finding is in contrast to observation of Nath et al ${ }^{[8]}$ where they found Diabetes Mellitus to be a significant risk factor for infected keratitis and ulceration.

As in most of the microbial profile analysis studies of corneal ulcer, in this study too anaerobic organisms could not be included due to lack of feasibility of maintaining anaerobic culture system.

\section{CONCLUSION}

The prevalence of infected corneal ulcer found in this hospital-based study in Southern part of Assam is 53.5\%. Microbial aetiology of corneal ulcer cases included fungal pathogens in $90 \%$ cases. Predominant fungal isolates were Aspergillus species, Fusarium species, Curvularia species, Sarocladium species, Penicillium species. Bacterial pathogens consisting of Pseudomonas aeruginosa and Staphylococcus aureus were detected in remaining $10 \%$ of the cases. Trauma by vegetative matter has been the most common predisposing factor for corneal ulceration and subsequent fungal infection. Higher numbers of cases of infected corneal ulcer were found in harvesting season and in patients associated with agricultural practice as they are more prone to exposed to fungal spores due to vegetative matter induced injury. Diabetes mellitus was not found to be a significant risk factor in cases of mycotic ulcers. Thus, selecting and initiating the appropriate antifungal and antibacterial drugs for empirical therapy after sending corneal scrap materials for microbiological examination will help in proper management and reduce morbidity due to this sight threatening condition.

\section{ACKNOWLEDGEMENT}

1. Dr. Debadatta Dhar Chanda, Professor \& HOD, Silchar Medical College \& Hospital, Silchar, Assam

2. Dr. Pinky Lahon. Demonstrator of Microbiology, Tezpur Medical College \& Hospital, Tezpur Assam.

Conflict of Interest: None of the two authors had conflict of interest in matters related to financial gain.

Financial Support / Grant: None

\section{Ethical Approval: Approved}

\section{REFERENCES}

1. Derek Y Kunimoto, Savitri Sharma, Prashant Garg, Usha Gopinathan, David Miller, Gullapalli N Rao, Corneal ulceration in the elderly in Hyderabad, South India, Br Journal of Ophthalmology 2000;84,54-59

2. Gopinathan U, Garg P, Fernandes $M$, Sharma S, Athmanathan S, Rao GN. The epidemiological features and laboratory results of fungal keratitis: a 10-year review at a referral eye care center in South India. Cornea 2002; 21:555-9.

3. Whitcher JP, Srinivasan M, Upadhyay MP. Corneal blindness: A global perspective. Bull World Health Organ 2001;79(3):21421

4. Lim AS. Mass blindness has shifted from infection (onchocerciasis, trachoma, corneal ulcers) to cataract. Ophthalmologica 1997; 211:270.]

5. Gonzales CA, Srinivasan M, Whitcher JP, Smolin G. Incidence of corneal ulceration in Madurai district, South India. Ophthalmic Epidemiol 1996; 3:159-66

6. Leck AK, Thomas PA, Hagan M, Kaliamurthy J, Ackuaku E, John M, et al. Aetiology of suppurative corneal ulcers in Ghana and south India and epidemiology of fungal keratitis. $\mathrm{Br} J$ Ophthalmol 2002; 86:1211-1215.

7. Bakshi R, Rajagopal R, Sitalakshmi G, Sudhi R, Madhavan H, Bagayalakshmi R. Clinical and Microbiological Profile of Fungal Keratitis: A 7 Year Study at a Tertiary Hospital in South India. Cornea Session III; AIOC 2008 Proceedings: 207209) 
8. Nath R, Baruah S, Saikia L, Devi B, Borthakur AK, Mahanta J. Mycotic corneal ulcers in upper Assam. Indian J Ophthalmol 2011; 59:367-71.

9. Tapan Gogoi, Sikha Deori, Shilpa Gupta, Current Trend of Corneal Ulcer in Patients Attending a Tertiary Healthcare Centre. International Journal of Science and Research; Vol 5; Issue 7, 68-71

10. Sanjeev Das, Jayashree Konar, Bacteriological profile of corneal ulcer with references to Antibiotic susceptibility in a tertiary care hospital in West Bengal Journal of Dental and Medical Sciences, Volume 11, Issue 6 (Nov.- Dec. 2013), PP 72-75

11. Rajesh Somabhai Katara, Nilesh Dhanjibhai Patel, and Mala Sinha, A Clinical Microbiological Study of Corneal Ulcer Patients at Western Gujarat, India; Acta Medica Iranica, Vol. 51, No. 6 (2013)

12. Kanishtha Sharma, Sonia Mehta, Varsha A Singh, Shivya Jamwal, Tanya Sharma, Prafulla Kumar Manjhi. A profile of corneal ulcers -2 years study from rural hospital, Haryana. Journal of Dental and Medical Sciences Volume 13, Issue 11 Ver. VII (Nov. 2014), PP 94-97

13. Anup K. Ghosh. Amit Gupta. Shivaprakash M. Rudramurthy. Saikat Paul. Vinay Kumar Hallur. Arunaloke Chakrabarti, Fungal Keratitis in North India: Spectrum of Agents, Risk Factors and Treatment; Mycopathologia DOI 10.1007/s11046-0160042-3
14. Dr. C. Senthil Vadivu, A study on bacterial, fungal and parasitic agents in infectious keratitis patients due to trauma in a tertiary care ophthalmic hospital, dissertation submitted to the Tamil Nadu Dr. M.G.R. Medical University, Chennai, Tamil Nadu for the - M.D. Degree (Microbiology)

15. K. Lakshmoji Naidu, Bhavani P.Srinivas, A Study On Clinical And Microbiological Evaluation Of Corneal Ulcers In GGH, Kakinada During 2013-2015, International Journal of scientific research and management (IJSRM), Volume3 Issue 4Pages 2564-2570, 2015

16. Ranjini CY, Vandana VW. Microbial profile of corneal ulcers in a tertiary care hospital in South India. J Ophthalmic Vis Res 2016; 11:363-7

17. Gandhi S, Shakya DK, Ranjan KP, Bansal S. Corneal ulcer: A prospective clinical and microbiological study. Int J Med Sci Public Health 2014; 3:1334-1337.

18. Mehta S, Mehta M. Clinical and Microbiological Profile and Treatment Outcome of Infective Corneal Ulcers: A Study in Central India. Int J Sci Stud 2017;4(12):234-24

How to cite this article: Bhattacharjee B, Chakravarty A. A study on microbial profile of corneal ulcer cases in a tertiary health care centre of Southern Assam. International Journal of Research and Review. 2021; 8(7): 228-235. DOI: https://doi.org/10.52403/ijrr.20210732 